\title{
EDITORAS EN CIERNES. EL ESPÍRITU EMPRESARIAL DE LAS LLAMADAS ESCRITORAS ISABELINAS ${ }^{1}$
}

\author{
HENRIETTE PARTZSCH \\ University of Glasgow
}

\begin{abstract}
Este artículo propone un abordaje interdisciplinario al estudio de las llamadas escritoras del canon isabelino para fomentar una mayor comprensión de su impacto en la cultura contemporánea. Los casos de Ángela Grassi, Faustina Sáez de Melgar, Joaquina García Balmaseda y Pilar Sinués de Marco muestran que su uso táctico del bricolage (Michel de Certeau) las sitúa en los intersticios de la autoría, el mundo de la edición y la industria de la moda. Iluminar la relación compleja entre su práctica de la autoría y sus polifacéticas iniciativas comerciales sería otro reto para la nueva biografía histórica.
\end{abstract}

PALABRAS CLAVE: emprendimiento, revistas de moda, España, siglo XIX.

\section{Editors in the Making: The Enterprising Spirit of Spain's Mid-Nineteenth Century Women Writers}

This article argues for an interdisciplinary approach to the study of the so-called escritoras isabelinas, Spain's mid-nineteenth century women writers, in order to develop a fuller understanding of their intervention in contemporaneous culture. The cases of Ángela Grassi, Faustina Sáez de Melgar, Joaquina García Balmaseda and Pilar Sinués de Marco show that their tactical use of bricolage (Michel de Certeau) situates them at the interstices of authorship, the publishing sector and the fashion industry. The complex relations between their authorship and their multiple commercial initiatives would be best captured from the perspective of the new historical biography.

KET WORDS: entrepreneurship, fashion magazines, Spain, nineteenth century.

Aunque se dice que cada libro y cada autor tienen su propio destino, la fortuna de las escritoras que empezaron a construirse una carrera profesional durante las dos últimas décadas de la época isabelina (1843-1868) se ha transformado más bien en una trayectoria colectiva. Después de disfrutar de un notable éxito individual, cayeron en un olvido compartido para ser nuevamente rescatadas hacia finales del siglo Xx gracias a los esfuerzos de pioneros historiadores de la literatura y de la

\footnotetext{
${ }^{1}$ Este trabajo se inscribe en el ámbito del Proyecto de Investigación "Prácticas culturales y esfera pública: Editoras españolas y latinoamericanas contemporáneas” (FFI2016-76037-P).
}

Partzsch, Henriette (2019), "Editoras en ciernes. El espíritu empresarial de las llamadas escritoras isabelinas", Lectora, 25: 77-90. ISSN: 1136-5781 D.O.I.: 10.1344/Lectora2019.25.4, Henriette.Partzsch@glasgow.ac.uk

Recepció: 15 de desembre de 2018 - Acceptació: 15 d'abril de 2019 
cultura, como por ejemplo Alicia Andreu, Alda Blanco, Lou Charnon-Deutsch, Ínigo Sánchez-Llama y Carmen Simón Palmer. La recuperación interpretativa se centraba en el análisis del discurso de la domesticidad plasmado en muchas contribuciones de Ángela Grassi (1823-1883), Faustina Sáez de Melgar (1834-1895) o Pilar Sinués de Marco (1835-1893), por nombrar solo a las más estudiadas. Con ello, se hacía hincapié en lo que Ginger y Lawless han bautizado como la tríada interpretativa de nación, género y representación (2018: 3), para intentar resolver los problemas de lectura planteados por unos textos que difícilmente se dejaban integrar en la crítica literaria al uso. Ese enfoque, una contextualización histórica guiada por las pautas de la lectura detallada, forma el fundamento del consenso académico actual acerca de las autoras en cuestión, según el cual, en resumidas cuentas, estamos ante un grupo de escritoras comprometidas con la escritura doméstica (Labanyi, 2017: 56-57).

Paradójicamente, el éxito del rescate bajo esas premisas ha contribuido a una nueva forma de invisibilidad para estas escritoras, ya que la etiqueta colectiva de "escritoras del canon isabelino" (Sánchez-Llama, 2000) se ha convertido en una manera sucinta para referirse en general a aquellas autoras decimonónicas cuyas obras siguen situándose en los márgenes del canon de lectura actual. Ocultas a plena vista, es necesario estudiar las prácticas vivenciales y profesionales de las isabelinas para empezar a entender su importante aportación a la cultura literaria de su tiempo, un enfoque que han adoptado recientes proyectos sobre la autoría de las mujeres (Fernández, 2015, 2017) y que corresponde a una tendencia reciente en los estudios culturales hispánicos que tiene por objetivo llegar a una comprensión matizada de dinámicas culturales complejas (Labanyi, 2012: 229). También la presente contribución se inscribe en esta perspectiva al analizar el protagonismo de las escritoras isabelinas no tanto desde su plasmación textual, sino como una intervención consciente en busca de oportunidades para transformar su pasión por las Letras en una propuesta económica sostenible, una dinámica que las convertiría en editoras en ciernes.

La importancia fundamental que tiene la vertiente económica del desempeño profesional de las isabelinas se debe a su posición social. Como revela un breve vistazo a sus biografías, en su mayoría estaban relacionadas con la clase media baja- y parece que ninguna de ellas podía contar con la seguridad de un sólido patrimonio familiar, una ventaja de la que gozaba por ejemplo Emilia Pardo Bazán (Grupo de Investigación La Tribuna, 2009) y Julia Codorniu, como documenta en el presente monográfico Turc-Zinopoulos. Seguí Collar, también en estas páginas de Lectora, evidencia que Faustina Sáez venía de una familia de pequeños terratenientes asentada en Villamanrique de Tajo y, aunque su matrimonio con Valentín Melgar Chicharro en 1855 le facilitara la mudanza a Madrid, los ingresos de la familia dependían del rendimiento de unas especulaciones mineras en Guadalajara

78

Lectora, 25 (2019): 77-90. ISSN: 1136-5781 D.O.I.: 10.1344/Lectora2019.25.4 
que poseía Valentín Melgar y distaban de ser copiosos o simplemente previsibles. Aparentemente el marido de Sáez solo tuvo empleos más estables a partir de 1867 -es decir, después de la primera desaparición del gran proyecto editorial de Faustina, la revista La Violeta (1862-1866), ingresando en el servicio público en 1868.

El caso de Pilar Sinués resulta más complejo. Su colega Ángela Grassi afirma en El Correo de la Moda que era "hija de nobles padres" (Grassi, 1862: 84), pero su semblanza biográfica solo hace hincapié en el hecho de que la abuela materna de Sinués hubiera vivido en el entorno real, una experiencia que le habría permitido pasar los modales de la Corte a la pequeña Pilar durante su primera infancia. Según la misma fuente, el padre de Pilar, Pedro Sinués y Yoldi, era empleado de la Administración Militar, por lo que la familia tuvo que mudarse a Burgos cuando Pilar tenía doce años. Parece que Pedro Sinués terminó sus días como oficial de la dirección de correos de Zaragoza, ya que el Montepío le adjudicó a su viuda, Flora Navarro, una pensión de 950 pesetas anuales en 1876 bajo este concepto (Diario Oficial de Avisos de Madrid, 26 noviembre 1876).

El resumen biográfico de Grassi indica que Pilar recibió una educación de señorita, pasando tres años con una tía materna en el convento zaragozano de Santa Rosa antes de ingresar como pensionista en otro colegio, donde estudió dibujo, francés y taquigrafía. Es posible que la mudanza familiar a Castilla pusiera fin a su educación -Grassi evoca a la adolescente "atenta solo a ayudar a su madre en sus quehaceres domésticos"-, hasta que una "enfermedad de decadencia” hizo necesario el regreso de la joven Pilar a Zaragoza, donde dedicaba un esfuerzo continuo a la literatura y desde donde finalmente consiguió sus primeras publicaciones. Es interesante observar que Grassi menciona "la amante aprobación de su familia" (Grassi, 1862: 85) hacia su desempeño literario, frente al tópico de la hostilidad del entorno familiar hacia las literatas noveles isabelinas. Como en el caso de Sáez de Melgar, el matrimonio, que Grassi fecha en 1855, le brindó a Sinués la oportunidad de trasladarse a Madrid. Contrariamente a Valentín Melgar, José Marco, el marido de Sinués, formaba parte de los círculos literarios de la capital como director de la revista La España Musical y Literaria. Además, era funcionario público (Romero Tobar, 2014: 5).

La extracción de Ángela Grassi y Joaquina García Balmaseda (Madrid, 18371911) es algo diferente, visto que ambas tenían una estrecha relación con el teatro y de ahí una experiencia más directa con el desempeño profesional. Según Simón Palmer (1991: 335), Grassi, la hija de un músico italiano, estudió música y obtuvo el título de maestra; durante los últimos quince años de su vida fue directora de la veterana revista El Correo de la Moda. No obstante, una biografía de la autora escrita por López de la Vega y publicada en 1879 en la revista Cádiz, dirigida por Patrocinio de Biedma, soslaya esta formación al mencionar solamente las lecturas que durante su infancia le habría proporcionado su madre. El golpe de teatro con 
el que Grassi entró en la escena literaria, el estreno de su drama Crimen y expiación en 1842 en el Teatro de Santa Cruz, lugar de trabajo de su padre, indica que aunque viviera "sumamente recogida", conocía bien el mundo teatral, ya que un drama que funciona en las tablas no se puede escribir "casi jugando", como afirma López de la Vega (1879: 130, 131). Respecto a García Balmaseda, no hay lugar a dudas de que la literata conocía el teatro de primera mano. De origen humilde, trabajó como actriz antes de emprender una larga carrera en la prensa, que la llevó a dirigir en 1864 la sección de literatura del diario La Correspondencia y, después de la muerte de Grassi, El Correo de la Moda (1851-1893) (Thion Soriano-Mollá, 2011).

Las cuatro escritoras presentadas, por lo tanto, no podían permitirse el lujo de escribir por escribir, pero tampoco se planteaban la actividad de publicar como una herramienta al servicio de un determinado movimiento, tal como lo hacían por ejemplo las gaditanas María Josefa Zapata (1822-?) y Margarita Pérez de Celis (1840?-1882) con su serie de Pensiles (1856-1866) inspirada en el pensamiento de Fourier (Espigado Tocino, 2008) o la espiritista Amalia Domingo Soler con La Luz del Porvenir (1879-1899). Para las literatas del canon isabelino, se trataba de combinar su pasión por las Letras con la necesidad de construir una actividad económicamente viable. Tuvieron que transformarse en agentes económicas de clase media, un reto al que se enfrentaban aparentemente con mucha lucidez, aunque lo comentaran públicamente solo en contadas ocasiones.

Una de esas ocasiones se encuentra en un pasaje que pertenece a una serie de ensayos (Partzsch, 2016) que Pilar Sinués publicó sobre la moda y la situación de las mujeres en diferentes países en La Moda Elegante Ilustrada en 1865-1866. En su análisis contrastado resuenan también viejos estereotipos y debates que acompañan los desequilibrios de poder entre naciones, pero también evidencia curiosidad y conocimiento acerca de las prácticas que se relacionan con la industria de la moda en su dimensión trasnacional. El pasaje reza así:

En España [a la mujer] le están vedados muchos caminos, muchos modos de ganarse la vida, que tiene en el estrangero: relegada á las ocupaciones rutinarias de la casa, su imaginacion activa se ocupa solo de idear galas y algunas veces - ¡con dolor lo digo! - se ocupa tambien de idear medios de adquirirlas, aunque no sean los mas honrosos. En España, la muger no tiene los mostradores, ni la pintura, ni la música para vivir: las artes y la industria no le abren ningún camino, porque los hombres los recorren todos - no siempre tampoco con la mejor fortuna- y le dicen á la débil mitad del género humano:

—Apártate! No aspires á lo que es mio! ¿Cómo lo he de partir contigo si no tengo bastante para mi?

80

Lectora, 25 (2019): 77-90. ISSN: 1136-5781 D.O.I.: 10.1344/Lectora2019.25.4 
Queden, pues, solo para la muger las faenas domésticas y calcular de qué modo podría aumentar sus atractivos físicos, único mérito que se le concede, pues los intelectuales pocos quieren reconocerlos y apreciarlos.

Esta falta total de ocupacion mental es, sin duda, la que hace que la muger en España salga mucho mas de su casa que en ninguna otra parte. (Sinués de Marco, 1865: 373; ortografía original)

Es interesante que la acusación articulada en este pasaje se le escapara de la pluma a Pilar Sinués, puesto que la zaragozana suele considerarse la escritora más asociada con el ideologema del ángel del hogar, debido a que publicó un libro (1859) y una revista (1864-1869) con este título. Sinués parte del lugar común de la mujer como consumidora frívola, percibida como amenaza del orden doméstico, para luego llamar la atención sobre el contexto social en que se produce el temido fenómeno del "exceso femenino", por citar el título de un trabajo de Alison Sinclair (2011). Según Sinués, la causa es la falta de oportunidades para integrarse en el mercado laboral, condición imprescindible para desarrollar un sentido de responsabilidad económica como las mujeres en Francia: "En una palabra, la muger en Francia sabe ganar dinero, y, por lo mismo, sabe economizarlo y gastarlo bien" (Sinués de Marco, 1866: 3; ortografía original).

De esta manera, el problema que la autora identifica no radica en una supuesta condición natural de las mujeres que las incapacitara para el desempeño profesional, sino en la fragilidad de la economía española: para los que ya están compitiendo en el mercado laboral, una creciente incorporación de las mujeres a la población activa representaría una amenaza de su propia posición. El hecho de que la publicación del ensayo de Sinués coincidiera prácticamente con el estallido de la burbuja ferroviaria y la resultante crisis financiera proporciona una perfecta ilustración de lo volátil que era la situación económica, también en el sector editorial (Martínez Martín, 2003: 605). Con ello, resulta evidente que Sinués entendía que los mecanismos del mercado capitalista penetraban en el santuario doméstico burgués afectando a la relación entre hombres y mujeres, pero articulándose de una manera específica en esta clase social. Mientras que activistas como Margarita Pérez de Celis denunciaban la brecha salarial entre hombres y mujeres en el sector manual, ya que resultaba indiscutible la necesidad del trabajo femenino en el contexto obrero (Pérez de Celis, 1864), en el ensayo citado Pilar Sinués intentaba normalizar la idea de salidas profesionales para mujeres de clase media gracias a la comparación con la situación en el país vecino. Sinués habla del comercio, del ámbito de las artes decorativas aplicadas y de la música como áreas con potencial para la profesionalización femenina. El denominador común de estos empleos era que no requerían estudios superiores. Desde nuestra perspectiva actual, esta limitación puede parecer una renuncia, estratégica o de convicción, a ocupar puestos 
destacados en la sociedad y reivindicar cambios más fundamentales. No obstante, en la España isabelina el pragmatismo de esa visión podía abrir un horizonte de progreso real.

La reivindicación matizada entroncaba con las prácticas educativas, con un currículum encauzado hacia las habilidades de adorno que estudiaban bastantes niñas de clase media, pero ampliaba su campo de aplicación, apoyándose en la fuerza del ejemplo y de los hechos cumplidos. En retrospectiva, esa táctica resultó bastante eficaz para obtener acceso al mercado laboral en todas sus dimensiones, profesiones liberales incluidas. Efectivamente, fue la insistencia en dar un paso tras otro lo que permitía a las primeras españolas convertirse en médicos profesionales (Flecha García, 1996: 75-86). El continuo goteo de solicitudes, basado en el aprovechamiento de lagunas o ambigüedades legales y el apoyo de compañeras y compañeros de viaje, iba creando antecedentes hasta que en 1910 la legislación normalizara la presencia de las mujeres en las universidades.

En el ensayo citado, Sinués no menciona la escritura, pero el desarrollo de esta habilidad con vistas profesionales se basaba en principios parecidos a los que la autora sugiere con respecto al arte y la música. Se trataba de usar una destreza aceptada, con sus aspectos decorativos si pensamos en la función social de mucha poesía de la época, como el filo fino de la cuña para ganar acceso a un mundo profesional en el que la participación económica visible de las mujeres de clase media no estaba prevista. Por esa exclusión, y en términos estrictamente estructurales, las isabelinas pertenecían a un grupo débil, en términos de Michel de Certeau (1988): las escritoras de clase media carecían de un sitio propio y solo podían insinuarse en el sitio ya ocupado por el otro, sin la posibilidad de apoderarse de él en su totalidad. El control que ejercían sobre su entorno profesional quedaba limitado y a menudo este se mostraba reactivo, por lo que necesitaban analizar constantemente las circunstancias para intentar convertirlas en oportunidades, una cuestión de supervivencia para los débiles, quienes tienen que actuar tácticamente porque no tienen las seguridades necesarias para desarrollar una estrategia estable. Por ello, la actividad económica de las isabelinas se inclinaba hacia lo que De Certeau denomina bricolaje: la re-combinación y el uso creativo de elementos preexistentes. Eran usuarias de unas estructuras y prácticas económicas en transición, las manipulaban para sus propios fines cambiándolas en el proceso, en busca de la constelación propicia de varios elementos heterogéneos. La necesidad de equilibrar elementos heterogéneos crea, por supuesto, contradicciones. Como observa De Certeau (1988: xix), la síntesis intelectual de los elementos aprovechados en el bricolaje no se plasma en forma de discurso, sino en una manera de pensar y actuar: en la práctica.

Es precisamente esta dimensión práctica la que da coherencia a las carreras polifacéticas de las isabelinas. Se explica por un fenómeno que Léo-Paul Dana y

82

Lectora, 25 (2019): 77-90. ISSN: 1136-5781 D.O.I.: 10.1344/Lectora2019.25.4 
Michael Morris (2011) han estudiado con referencia a las minorías étnicas y que consiste en el hecho de que la ausencia de salidas profesionales establecidas, tal como la denuncia Sinués en el ensayo citado, suele despertar la iniciativa empresarial en los miembros más activos de grupos marginados. En este contexto, resulta significativo que la cita de Pilar Sinués aparezca en una revista de moda, el vehículo que daba a muchas mujeres la primera oportunidad de ver un texto suyo en letras de molde. No obstante, publicar textos en la prensa no ofrecía una perspectiva clara de ingresos regulares. El testimonio de Pardo Bazán sobre el estado de la cuestión durante los primeros años de su carrera literaria resume perfectamente la situación:

[M]i labor literaria, allá por los años de 1878, distaba tanto de prometerme ventajas económicas, que el importe de mi primer artículo que espontáneamente me pagó un editor (catalán por más señas) lo invertí en una sortija cintillo de brillantes, para conservar el recuerdo por si no volvía a repetirse el caso. (Grupo de Investigación La Tribuna, 2009: 55)

Ahora bien, la función de las revistas de modas y salones no se limitaba a facilitar el paso de escritora casera a autora publicada. Para las isabelinas más emprendedoras, cabeceras como El Correo de la Moda, La Moda Ilustrada Elegante, La Violeta o El Ángel del Hogar se convertían en plataformas flexibles y, por lo tanto, idóneas para ensamblar diferentes piezas de bricolaje en busca de perspectivas económicas y profesionales. Su gran interés radicaba en el carácter híbrido de las publicaciones al situarse en la encrucijada entre la autoría, el sector de la edición y una industria que formaba la vanguardia del capitalismo global.

Es difícil sobreestimar el juego que daba ese posicionamiento al creciente número de literatas involucradas en el negocio de las revistas. La combinación de elementos abría una amplia gama de oportunidades para el desempeño profesional, desde la escritura literaria sensu stricto hasta la colaboración comercial local, nacional y global, pasando por la edición de productos impresos afines y el aprovechamiento de las redes de contacto y de distribución de una de las industrias más avanzadas de la época. En este sentido, las revistas de moda ofrecían un espacio económico muy atractivo porque proporcionaban una salida para la escritura, al mismo tiempo que mitigaban el riesgo económico asociado con la publicación de literatura.

La diversificación del producto iba más allá del simple hecho de cubrir diferentes áreas de interés - moda, noticias de sociedad, literatura, ensayo- en una sola publicación. La estrecha relación de las revistas con el comercio de la moda se plasmaba por ejemplo en las recomendaciones de ciertas modistas, de tiendas o de productos específicos, que a veces incluso se podían comprar en las oficinas de la 
redacción. En la época parisina de La Violeta, Sáez convirtió esta práctica en un servicio de compras a distancia, como se desprende de la siguiente información para sus lectores:

La administración de La Violeta y sus varias redactoras, que desean sobre todo complacer á sus abonados y corresponsales, se ponen á la disposición de los mismos para cuantos encargos quieran confiarles respecto á compras, de cualquier género que sean; les basta interesarse por nuestro periódico, para tener derecho á que se sirvan y atiendan sus peticiones. (La Violeta, 1884: 2; ortografía original).

Es posible que Sáez aplicara al relanzamiento de La Violeta conocimientos e ideas que había adquirido en su actividad editorial como directora de París-Charmant/París-Charmant-Artístico (1881-1883), revista publicada por los grandes almacenes Saint-Joseph para el mercado hispanoamericano (Seguí Collar, en el presente volumen). Ya en Madrid, Faustina Sáez había llegado a acuerdos con otras empresas para atraer y fidelizar a los suscriptores de La Violeta (primera época). El ejemplo más destacado es la colaboración con el taller fotográfico del prestigiosísimo J. Laurent, un golpe de efecto que le permitió ofrecer vales para hacerse fotografiar a quienes abonaban la revista por un año entero; los suscriptores que no residían en Madrid podían pedir fotografías del catálogo de Laurent. Con ello, La Violeta y su directora aprovechaban la fascinación con la imagen que se plasmaba en la moda de las cartes-de-visite y la reputación que tenía Laurent como retratista de la Corte.

No obstante, el principio de colaboración sobrepasaba las iniciativas llevadas a cabo por algunas cabeceras individuales porque formaba el fundamento mismo del negocio de las revistas de moda, aunque la envergadura de la interdependencia no siempre fuera obvia. Como mínimo, las publicaciones tenían que incluir los codiciados figurines de París, un imperativo que las empujaba a buscar arreglos con publicaciones francesas; por ejemplo, La Violeta (primera época) solía recibir sus grabados de Les Modes Parisiennes (1843-1885). La colaboración de La Moda Elegante Ilustrada (1863-1923) con La Mode Illustrée (1860-1937) iba mucho más allá de comprar figurines, como demuestra la presencia continua de la firma de Emmeline Raymond, directora de la revista francesa entre 1860 y 1902, en la sección de información indumentaria de la publicación española (Partzsch, 2016: 284). Sin embargo, el elemento compartido más llamativo es la disposición de las páginas, caracterizadas por la integración de ilustraciones xilográficas en el cuerpo del texto. Este formato señala que ambas publicaciones formaban parte de una compleja red internacional de revistas inspiradas en o sindicadas con la famosa Der Bazar de Berlín, cuya influencia se hacía notar en toda Europa y las Américas,

84

Lectora, 25 (2019): 77-90. ISSN: 1136-5781 D.O.I.: 10.1344/Lectora2019.25.4 
hasta tal punto de que la revista afirmó en 1873 que era el periódico global más difundido (Van Remoortel, 2017: 269).

La posición peculiar que tenían las revistas de moda gracias a la conexión con el sector textil global añade otra dimensión al desempeño profesional de las isabelinas, también con respecto a la creación de redes de contacto: la necesidad imperiosa de conocer a socios comerciales potenciales, tener acceso a recursos o conocimientos y formar expectativas compartidas (Staber, 2011: 191-92). Queda bien establecido que durante la época isabelina las escritoras, en tanto que mujeres, tenían un acceso limitado o inexistente a muchos ambientes profesionales y sociales frecuentados por los hombres de Letras como el funcionariado y los casinos. La única manera de insinuarse en esos círculos vedados era tomar la vía indirecta, facilitada por mediadores que, o bien se interesaban activamente en las carreras de las literatas, como el infatigable Juan Eugenio Hartzenbusch, o bien tenían relaciones familiares o de amistad con las escritoras y sus familias. Como explica Noël Valis, "en cierto sentido todo empieza en su 'gabinete', en el hogar, con los lazos familiares y de amistades que tejen el diseño cotidiano de sus vidas, para luego adaptarse a nuevos modelos ideológicos y desplazarse a escenarios más públicos" (Valis, 2015: 103).

Por supuesto, las isabelinas se servían de las oportunidades ofrecidas por lugares de sociabilidad más abiertos al protagonismo de las mujeres. En Madrid, parece que el Liceo Piquer, un teatro privado creado por el escultor José Piquer y su mujer, Emilia Llull, jugaba un papel especialmente importante como lugar de encuentro (Freire López, 2008). Gracias a las frecuentes noticias acerca de las actividades del Liceo publicadas en La Violeta sabemos que en las actuaciones coincidían por ejemplo García Balmaseda y Grassi (Sáez de Melgar, 1864: 11). El hecho de que Emilia Llull de Piquer se encontrara entre los primeros contribuyentes a una colección caritativa organizada por la revista (La Violeta 104, 27 noviembre 1864) indica que había relaciones más estrechas entre las dos directoras. Ya en la década siguiente, Emilia Llull acogería también a Concepción Gimeno y una de sus obras teatrales (Simón Alegre, 2012). La función de escaparate cultural del Liceo Piquer para las isabelinas queda, por lo tanto, bien documentada, pero solo podemos especular si y cómo los contactos establecidos en las reuniones también contribuían a sus actividades de emprendedoras, por ejemplo a la hora de buscar financiación.

Ahora bien, a ese arraigo en instituciones del entorno cultural inmediato se añade la dimensión inter y transnacional característica de la industria de la moda, pero también del comercio del libro globalizado (Fernández, 1999). Esta constelación parece prefigurar una actitud empresarial que ganó tracción hacia finales del siglo Xx, la estrategia glocal que combina operaciones globales y locales y que, se supone, ofrece una escapatoria para la supervivencia de pequeñas y medianas 
empresas gestionadas por el/la propietario/a (Obrecht, 2011: 131). Es cierto que los dos pilares de lo local y lo global dejaron sus huellas en las publicaciones de moda decimonónicas. En el caso de La Violeta, es la sección de anuncios publicados la que marca el entretejimiento con el entorno comercial madrileño. Hay múltiples anuncios de una tienda de novedades situada en la calle Concepción Gerónima 5, en la inmediata vecindad de la redacción, sita en la calle Concepción Gerónima 13, que en dos ocasiones recurrió a la revista también para buscar una oficiala mayor.

El anuncio para un sanatorio en Cuba recuerda el contexto global en que se insertaba el negocio de La Violeta. El hecho de que el texto publicitario se dirigiese especialmente a viajeros llama la atención sobre el influjo de las estructuras coloniales en las redes de contactos, un cauce que Sáez usó probablemente en sus intentos para entrar en el mercado filipino (Partzsch, 2018: 202). No obstante, las relaciones globales suelen despuntar más en otras secciones de la revista. El discurso de algunas columnistas de moda evoca una Jauja capitalista en la que los productos del mundo entero se ponen al servicio de las consumidoras (Partzsch, 2016: 281-82), mientras que la información miscelánea permite revelar aspectos más concretos de las colaboraciones internacionales, como por ejemplo los lazos que tenía La Violeta con el periódico y la imprenta La Concordia en Caracas a través de su propietario Evaristo Fombona (Partzsch, 2018: 201-2), una relación que explica que Sáez escribiera dos entregas de la "Revista de Madrid" en forma de carta a una supuesta prima habitante en "el país tropical” (Sáez de Melgar, 1866a: 281), aprovechando textos que, según la autora, también se publicaron en La Concordia (Sáez de Melgar, 1866b: 302).

La colaboración con Caracas ilustra el potencial que tenía la "variopinta red de progresiva constitución [que] acompaña la dificultosa emergencia de un mercado nacional y la conquista del mercado hispanoamericano" (Botrel, 2003: 609). Las específicas constelaciones de esta red determinaban la difusión de un producto impreso a los niveles local, nacional y global. La Violeta tenía una buena presencia en las tiendas de destacados libreros e impresores madrileños, como el conocido negocio de Bailly-Baillière. Fuera de Madrid, una red de libreros corresponsales completaba la suscripción individual, tanto en España como en las Américas (Partzsch, 2016: 197, 199-200). De particular interés son los nombres que parecen indicar conexiones personales y profesionales transatlánticas, como en los casos del librero e impresor Antonio Pérez Dubrull y de la librería Ambos Mundos de Medina Hermanos. Son los dos negocios madrileños que publicaron con mayor regularidad anuncios en La Violeta, al mismo tiempo que las librerías Medina hermanos en Buenos Aires y Juan Pérez Dubrull en Santiago de Cuba ofrecían servicios de corresponsalía para los suscriptores americanos de la revista. Al facilitar la

86

Lectora, 25 (2019): 77-90. ISSN: 1136-5781 D.O.I.: 10.1344/Lectora2019.25.4 
redifusión de contenidos, el arreglo entre La Violeta y La Concordia profundizó en este modelo de colaboración.

Las isabelinas se desempeñaban, así pues, en un marco muy específico con respecto a las condiciones de producción, difusión y valoración de textos. Este marco las sitúa como objeto de estudio en el intersticio de dos disciplinas, los estudios literarios y la historia del empresariado. El abordaje desde solo una de las dos crea, por consecuencia, tensiones con las categorías básicas que determinan el análisis. En tanto profesionales del mercado de los impresos, las isabelinas no corresponden ni a la idea del autor que se debe ante todo a la verdad literaria, ni al personaje del editor que tiende puentes entre el autor y el público lector; más bien fusionan características de ambos roles, creando en el proceso nuevas maneras de intervenir en la cultura literaria. Por otro lado, sus iniciativas empresariales no las convierten en emprendedoras en el sentido de las definiciones clásicas, según las cuales estamos ante un individuo que funda, posee y lidera un negocio, si es posible innovando en el proceso. Aunque las cuatro literatas presentadas llegaron a ocupar cargos destacados en una variedad de negocios, su estatus legal y las condiciones culturales impedían que tuvieran las riendas en la mano oficialmente.

El alcance del "bricolaje táctico" de las isabelinas en el sector cultural sólo se revela si, siguiendo a Baumol, entendemos el empresariado como un proceso complejo que no sigue un desarrollo lineal y en el que los emprendedores "entran y salen de los campos de actividad que normalmente se reconocen como empresariales, intercambiándolos por otras actividades que también requieren un espíritu emprendedor pero que muchas veces se alejan de la producción de bienes y servicios" (Baumol, 2011: 53). ${ }^{2}$ La complejidad de esa forma discontinua de intervenir en la sociedad no se deja captar ni mediante la interpretación de textos individuales ni mediante el estudio de iniciativas comerciales específicas. Aunque ambos enfoques aportan información imprescindible, solo pueden ofrecer instantáneas. Contar la historia del desarrollo profesional de las isabelinas será, pues, otro reto para el abordaje desde la nueva biografía (Gallego Franco y Bolufer Peruga, 2016).

\section{REFERENCIAS BIBLIOGRÁFICAS}

Baumol, William (2011), "Economics and Entrepreneurship", World Encyclopedia of Entrepreneurship, Léo-Paul Dana (ed.), Cheltenham y Northampton, Edward Elgar: 53-64.

2 " $[\mathrm{T}]$ hey move in and out of the activities usually recognized as entrepreneurial, exchanging them for other activities that also require enterprising talent but are often distant from the production of goods and services" (mi traducción). 
Botrel, Jean-François (2003), "La difusión del libro", Historia de la edición y de la lectura en España 1472-1914, Víctor Infantes de Miguel, François López y JeanFrançois Botrel (dirs.), Salamanca, Fundación Ramón Sánchez Ruipérez: 609618.

Certeau, Michel de (1988), The Practice of Everyday Life, Steven F. Rendall (trad.), Berkeley, University of California Press.

Dana, Léo-Paul y Michael Morris (2011), "Ethnic Minority Entrepreneurship", World Encyclopedia of Entrepreneurship, Léo-Paul Dana (ed.), Cheltenham y Northampton, Edward Elgar: 149-157.

Espigado Tocino, Gloria (2008), "La Buena nueva de la mujer profeta: Identidad y cultura política en las fourieristas Ma Josefa Zapata y Margarita Pérez de Celis", Pasado y memoria. Revista de historia contemporánea, 7: 15-33

Fernández, Pura (1999), "La editorial Garnier de París y la difusión del patrimonio bibliográfico en castellano en el siglo XIX", Tes philies tade dora. Miscelánea léxica en memoria de Conchita Serrano, Madrid, CSIC: 603-612.

-(ed.) (2015), No hay nación para este sexo. La Re(d)pública trasatlántica de las Letras: Escritoras españolas y latinoamericanas (1824-1936), Madrid y Frankfurt, Iberoamericana-Vervuert.

-(ed.) (2017), “'Por ser mujer y autora'... Identidades autoriales de escritoras y artistas en la cultura contemporánea", Insula. Revista de letras y ciencias humanas, 841-842: 2-7.

Flecha García, Consuelo (1996), Las primeras universitarias en España, Madrid, Narcea.

Freire López, Ana María (2008), "El Liceo Piquer: Un ámbito para la convivencia de las artes en la segunda mitad del siglo XIX", La literatura española del siglo XIX y las artes, Jean-François Botrel et al. (eds.), Barcelona, PPU: 129-140.

Gallego Franco, Henar y Mónica Bolufer Peruga (eds.) (2016), ¿Y ahora qué? Nuevos usos del género biográfico, Barcelona, Icaria.

Ginger, Andrew y Geraldine Lawless (2018), "Introduction", Spain in the Nineteenth Century: New Essays on Experiences of Culture and Society, Andrew Ginger y Geraldine Lawless (eds.), Manchester, Manchester University Press: $1-12$.

Grassi, Ángela (1862), “María del Pilar Sinués de Marco”, El Correo de la Moda, 443: 84-86.

Grupo de Investigación La Tribuna (2009), "La riqueza de Emilia Pardo Bazán. Una aproximación a su estudio", La Tribuna, 7: 37-79.

Labanyi, Jo (2012), "Doing Things: Emotion, Affect, and Materiality", Journal of Spanish Cultural Studies, 11 (3-4): 223-233.

88

Lectora, 25 (2019): 77-90. ISSN: 1136-5781 D.O.I.: 10.1344/Lectora2019.25.4 
-(2017), "Afectividad y autoría femenina. La construcción estratégica de la subjetividad en las escritoras del siglo XIX”, Género y subjetividad en la España del siglo XIX (un diálogo entre la historia y la literatura), Mónica Burguera (ed.), Espacio, tiempo y forma, 29: 41-63.

López de la Vega, Dr. [¿José?] (1879), “Escritoras contemporáneas. Doña Ángela Grassi”, Cádiz, 17: 130-132.

Martínez Martín, Jesús A. (2003), "Editores y empresas editoriales”, Historia de la edición y de la lectura en España, 1472-1914, Víctor Infantes de Miguel, François López y Jean-François Botrel (dirs.), Salamanca, Fundación Ramón Sánchez Ruipérez: 601-608.

Obrecht, Jean J. (2011), “Environment for Entrepreneurship”, World Encyclopedia of Entrepreneurship, Léo-Paul Dana (ed.), Cheltenham y Northampton, Edward Elgar: 122-139.

Partzsch, Henriette (2016), “'Venid, elegantes’: Seducción, información y control en las crónicas de modas españolas durante los años 1860", Redes de Comunicación. Estudios sobre la prensa en el mundo hispanohablante, Yvette Bürki y Henriette Partzsch (eds.), Berlín, Frank \& Timme: 281-303.

-(2018), "How to Be a Cultural Entrepreneur", Spain in the Nineteenth Century: New Essays on Experiences of Culture and Society, Andrew Ginger y Geraldine Lawless (eds.), Manchester, Manchester University Press: 191-215.

Pérez de Celis, Margarita (1864), "Injusticia social”, La Crónica de Gerona, 3-4: 12.

Romero Tobar, Leonardo (2014), "María Pilar Sinués, de la provincia a la capital del Reino", Arbor, 190 (767), 01/09/2018. <http://dx.doi.org/10.3989/arbor. 2014.767n3012>

Sáez de Melgar, Faustina (1864), "Revista de teatro", La Violeta, 57: 10-11.

—(1866a), "Revista de Madrid", La Violeta, 197: 284-86.

-(1866b), "Revista de Madrid", La Violeta, 199: 300-302.

Sánchez-Llama, Íñigo (2000), Galería de escritoras isabelinas. La prensa periódica entre 1833 y 1895, Madrid, Cátedra.

Simón Alegre, Ana Isabel (2012), “Concepción Gimeno y el ocio teatral madrileño en 1873", Pasado, presente y porvenir de las humanidades y las artes IV, Zacatecas, Texere: 433-466.

Simón Palmer, Carmen (1991), Escritoras españolas del siglo XIX. Manual biobibliográfico, Madrid, Castalia.

Sinclair, Alison (2011), "Luxurious Borders: Containment and Excess in Nineteenth-Century Spain”, A Companion to Spanish Women's Studies, Xon de Ros y Geraldine Hazbun (eds.), Woodbridge, Tamesis: 211-226. 
Sinués de Marco, Pilar (1865), "La mujer española. Carácter particular de la misma. Su educación. La moda en España”, La Moda Elegante Ilustrada, 47: 373-374.

-(1866), "La mujer francesa. Su carácter. Comparaciones. Consejos", La Moda Elegante Ilustrada, 1: 3-4.

Staber, Udo (2011), "Geographic Proximity in Entrepreneurship", World Encyclopedia of Entrepreneurship, Léo-Paul Dana (ed.), Cheltenham y Northampton, Edward Elgar: 188-197.

Thion Soriano-Mollá, Dolores (2011), “Joaquina García Balmaseda: Una escritora isabelina al servicio de la mujer”, Anales de literatura española, 23: 381-403.

Valis, Noël (2015), "Patronazgo masculino y visibilidad de las escritoras románticas españolas y norteamericanas", No hay nación para este sexo. La Re(d)pública transatlántica de las Letras: Escritoras españolas $y$ latinoamericanas (1824-1936), Pura Fernández (ed.), Madrid y Frankfurt, Iberoamericana-Vervuert: 83-109.

Van Remoortel, Marianne (2017), "Women Editors and the Rise of the Illustrated Fashion Press in the Nineteenth Century", Nineteenth Century Contexts, 39 (4): 269-295. 